Courant Mathematics and

Computing Laboratory

U.S. Department of Energy

\title{
The Bifurcation of Tracked Scalar Waves
}

J. Glimm, J. Grove, B. Lindquist,

O. A. McBryan and G. Tryggvason

Research and Development Report

Supported by the Applied Mathematical Sciences

subprogram of the Office of Energy Research,

U.S. Department of Energy under

Contract DE-AC02-76ER03077

Mathematics and Computers

May 1986 

UNCLASS IF IED

Courant Mathematics and Computing Laboratory
New York University

Mathematics and Computers

UC -32

$\mathrm{DOE} / \mathrm{ER} / 03077-272$

THE BIFURCATION OF TRACKED SCALAR WAVES

J. Glimm, J. Grove, B. Lindquist,

O. A. McBryan and G. Tryggvason

May 1986

Supported by the Applied Mathematical Sciences subprogram of the Office of Energy Research, U. S. Department of Energy under Contract No. $\mathrm{DE}-\mathrm{AC} 02-76 \mathrm{ER} 03077$ 


\title{
DISCLAIMER
}

Th1s report was prepared as an account of work sponsored by an agency of the United States Government. Ne1ther the Un1ted States Government nor any agency thereof, nor any of their employees, makes any warranty, express or Implied, or assumes any lep,al l1ab1l1ty or respons1b1l1ty for the accuracy, completeness, or usefulness of any Information, apparatus, product, or process disclosed, or represents that 1 ts use would not infrinfe privately owned rights. Reference herein to any specific commercial product, process, or service by trade name, trademark, manufacturer, or otherwise, does not necessarlly constitute or 1mply 1 ts endorsement, recommendation, or favoring, by the United States Government or any arency thereof. The views and op1nions of authors expressed here1n do not necessarlly state or reflect those of the United states Government or any agency thereof.

\author{
Printed in U.S.A.
}

Avallable from

Nat1onal Technical Information Service

U.S. Department of Commerce

5285 Port Royal Road

Springfield, VA 22161 
CONTENTS

\section{Page}

Abstract

1. Introduction

2. The Algorithm

3. Examples

4. Conclusion

References

Figures 



\title{
The Bifurcation of Tracked Scalar Waves
}

\author{
James Glimm 1,2,3 \\ John Grove 1, 2 \\ Brent Lindquist ${ }^{1}$ \\ Oliver A. McBryan 1, 3,4.5 \\ Gretar Tryggvason ${ }^{3}$ \\ Department of Mathematics \\ Courant Institute \\ New York University \\ New York, NY 10012.
}

\begin{abstract}
The dynamic evolution of tracked waves by a front-tracking algorithm may lead on either numerical or physical grounds to intersections of the waves. The correct resolution of these intersections is described locally by the solution of Riemann problems and requires a bifurcation of the topology defined by the tracked waves.

Here we describe an algorithm that is appropriate for the resolution of scalar tracked waves, such as material discontinuities, contact discontinuities in gas dynamics, or constituent concentration waves including oil-water banks in oil reservoirs. Even here the algorithm is not fully general, and the resolution of the intersections of an arbitrary set of curves in the plane for the above range of physical problems remains unsolved. However with the assumption that the set of intersections to be resolved is a small perturbation (resulting for example from a small time step in an evolution) of a valid, non-intersecting front, the algorithm seems to be general. In any case examples will be presented that show that complicated interfaces can be generated automatically from simple ones through successive bifurcations.
\end{abstract}

1 Supported in part by the Applied Mathematical Sciences subprogram of the Office of Energy Research, U. S. Department of Energy, under contract DE-AC02-76ER03077.

2 Supported in part by the Army Research Office, grant DAAG29-85-0188.

3 Supported in part by the National Science Foundation, grant DMS-83-12229.

4 Supported in part by ARO grant DAAG29-84-K-0130

5 Alfred P. Sloan Foundation Fellow. 


\section{Introduction}

Front tracking is a computational method based on nonlinear wave analysis. The nonlinear waves and their interactions are conceptual building blocks which serve to organize the computation. This method is characterized by high resolution solutions for problems with significant solution discontinuities, such as shock waves and material boundaries. In addition to its computational achievements, listed for example in $[1,2,3,4,5]$, the development of front tracking has lead to advances in the mathematical theory of nonlinear wave analysis and to the solution of a long standing problem for pure finite differences: the 20 year old Rachford problem of viscous fingering for immiscible displacement with capillary diffusion. [6] The present article is devoted to the solution of a problem which had been viewed as a major stumbling block for front tracking, namely the intersections of fronts with other fronts and with boundaries. The purpose of this article is to provide a solution valid for the case of tracked scalar waves. Partial results for vector wave (shock) interactions have been reported elsewhere. [1]

We distinguish between bifurcations in the computed approximate solution that arise on numerical grounds and do not have a counterpart in the exact mathematical solution of the underlying equations, and those that do correspond to bifurcations in the exact mathematical solutions of the underlying equations. In the first category are material interfaces and contact discontinuities. Within the limits of the governing equations (neglecting higher order effects such as diffusion and surface tension), such interfaces should come arbitrarily close and form filaments, without ever intersecting. Such filaments are numerically difficult to compute and usually of little importance, so that an approximate solution method that allows intersections to occur and then resolves them is acceptable. From a mathematical point of view, this means simply that convergence is not sought in the $L_{x}$ norm but in $L_{1}$ instead. The occurrence of these intersections may be associated in part with the order of accuracy of the time integration or with the size of the time step and in part with the nature of the unstable modes excited. For example the method of $[7,8]$ which uses a high order time integration scheme almost never develops actual crossing of the interfaces. The front tracking code used in our calculations will develop crossings for comparable problems in which unstable fingers dominate, though such crossings are relatively few in number. However the unstable jets with pinch nodes excited reported on here, lead to interface crossings on a very regular basis.

The other case, in which the bifurcation is a property of the exact mathematical solution, can occur when the tracked discontinuity curve represents a shock wave. We distinguish between vector shock waves such as shocks in the acoustic mode of compressible fluids, and scalar shocks such as those representing concentration or saturation fronts in oil reservoirs, adsorption problems, or chemically reacting fluids. The algorithm discussed in this paper is 
appropriate only for tracked scalar shock waves. A preliminary discussion of the bifurcation of vector shock waves is given in [1].

Modifications of the front may thus be necessary for both physical and numerical reasons. Algorithms which accomplish such tasks have been referred to as "untangling algorithms" by the authors. To avoid this problem, most front tracking codes use some version of the volume of fluid method. In the volume of fluid codes the front is not represented by continuous curves, as in our code, but is reconstructed from a marker function, which represents the amount of each fluid in each grid block. The resulting description of the front is of a lower quality than it is for front tracking as defined by us, see [9]. For example the volume of fluid method may cause numerical entrainment and fluid mixing. See [10,11] for a further discussion of interface methods and see $[9,3,4,12,5]$ for additional discussion of front tracking as used in this paper.

Over several years we have experimented with preliminary versions of untangle algorithms which have lead to the one described here. A related untangling problem in our code occurs in the generation of a finite element grid, used to solve elliptic equations. An initially rectangular grid is locally distorted by shifting some grid points along grid lines to adjacent front curves such that on the resulting grid the front lies solely along the boundaries of the finite elements [13]. In a complex front, extremely close curves cause an ambiguity for the grid point shifting. In such cases both the curves and grid are locally distorted to intersect at a common position. Here the curves intersect, although they do not cross, and these curve crossings have to be resolved before the elliptic solver is invoked. The algorithm to accomplish this is considerably simpler than the one discussed here, and has been described in [5].

\section{The Algorithm}

To establish the rules governing how an interface is to be untangled it is necessary to make some assumptions about the tangled interface. Before we state explicitly the restrictions we expect to hold for the evolution of the interface let us establish our terminology. (See [11] for a more complete description of the interface.) We restrict our discussion here to two spatial dimensions though the restrictions placed on the front evolution are equally valid in three dimensions. By a curve, we mean a piecewise linear, oriented, curve segment in $R^{2}$. The linear pieces are called bonds. The start and end points of a curve (which need not be distinct) are called nodes. In general several curves will start or end at a common node. The incoming curves for a given node are those that end at that node, while the outgoing curves are those that start there. An interface is a collection of curves and nodes. A proper, or untangled interface is one whose curves intersect (including self-intersections) only 
at their start or end points, the nodes. Otherwise the interface is said to be tangled. These points of interior curve intersection are called cross points. We consider two interfaces, an untangled interface $I^{0}$ given at the beginning of a time step and a possibly tangled interface $I^{1}$ defined at the end of the time step. The interface $I^{1}$ is connected to the interface $I^{0}$ by a linear homotopy, with parameter $t, 0<t<1$. We call this homotopy the time step homotopy. The interfaces $I^{0}$ and $I^{1}$ separately divide the plane $R^{2}$ into regions called components. The same is true for each of the intermediate interfaces $I^{\prime}$. If a given component of the $I^{1}$ interface does not connect continuously to a component of the $l^{0}$ interface it is called an unphysical component. Otherwise it is physical.

The main assumption of the untangling algorithm described here is that the curves do not cross each other in such a way that the resulting "tangled" interface $I^{1}$ is physically meaningful. This assumption simplifies the identification of interface tangling having occurred under the homotopy by ensuring that the tangle is captured in the form of crossed curves in the $I^{1}$ interface. Our explicit assumptions are:

A1. Each point on a curve in $I^{1}$ has crossed some other curve at most once during the last time step in which $I^{0}$ is displaced to $I^{1}$.

A2. Each unphysical component has on its boundary at least one cross point where certain curves of $I^{1}$ intersect.

A3. At the cross points the curves really cross each other, rather than merely touch.

A4. No nodes have crossed a curve during the time step.

Assumption A1 is really a limit on the size of a time step. A step that causes violation of assumption $\mathrm{Al}$ is too big and must be reduced. The restrictions implied by the other assumptions can be enforced by suitable preprocessing before the actual untangling algorithm is started. Assumption A2 excludes the possibility that a closed loop "inverts" itself. (See Fig. 1.) Such loops can be identified by comparing loop orientation at $t=0$ and $t=1$. Assumption $\mathrm{A} 3$ is included for completeness; the probabilities of a point lying exactly on another curve are obviously small. Nodes that have crossed a curve must also be identified in advance and a separate algorithm run first to untangle these node interactions. Preprocessing of this sort has been carried out to some degree in our computations.

The untangling procedure then consists of finding the cross points of the $I^{1}$ interface, and identifying the unphysical areas between curves that have crossed each other. The first step in the algorithm is to find the intersections or cross points. This is done in an efficient way described in [11]. Next the cross points are replaced by nodes. The resulting nodes are called non-homotopic $(\mathrm{NH})$ nodes, to distinguish them from the (homotopic) nodes of the interface $I^{1}$, which are connected by the homotopy to the nodes of the interface $l^{0}$. The (old) 
curves of $I^{1}$ that cross at these points are split, and the new curves resulting from the split are attached to the $\mathrm{NH}$ nodes. The main body of the untangling algorithm then consists of looping over these NH nodes, and determining which connected curves enclose an unphysical component. In doing so we are aided by several observations that are seen to follow immediately from the assumptions $\mathrm{A} 1$ to $\mathrm{A} 4$.

O1. Every $\mathrm{NH}$ node must have two incoming curves and two outgoing curves, dividing the area surrounding the $\mathrm{NH}$ node into four angular sectors. An incoming and outgoing curve can, however, be the same curve.

O2. One, and only one, of the four sectors surrounding an NH node is unphysical.

O3. If a curve starts and ends at the same $\mathrm{NH}$ node (i.e. is a loop), the two sectors outside the curve must be physical.

O4. The sector between two curves that each join a single $\mathrm{NH}$ node $n_{1}$ to distinct nodes $n_{2} \neq n_{3}$, must be physical if at least one of the nodes $n_{2}$ or $n_{3}$ is a homotopic node.

O5. An unphysical sector must be bounded by either:

(a) a single curve that starts and ends at the same $\mathrm{NH}$ node.

(b) two curves that go from a common node $n_{1}$ to another common node $n_{2}$, one of which must be a NH node.

We will not prove in detail any of these observations, although they are perfectly general conclusions from our assumptions. Observation $\mathrm{O} 1$ is trivial, since a cross consists of two bonds belonging either to different curves or the same curve and after an $\mathrm{NH}$ node has been created both bonds are split and attached to the node. Observation $\mathrm{O} 2$ is valid from restrictions $A 1$ and $A 3$. Observation $O 3$ follows from observation $O 2$, since for a curve that starts and ends at the same NH node, the component exterior to this loop forms two of the sectors adjacent to the $\mathrm{NH}$ node. However, from observation $\mathrm{O} 2$ one, and only one, can be unphysical. Observation $\mathrm{O} 4$ is a consequence of assumption A4.

The implementation of the untangling algorithm consists of two parts. The first part identifies those physical components that can be unambiguously determined as such from the topology of the interface $I^{1}$. For those $\mathrm{NH}$ nodes that then have only one remaining unidentified sector, this must be the unphysical one and the curves bounding it are therefore deleted. The second part of the algorithm deals with nodes that have two or more adjacent areas that could possibly be unphysical, but can not be identified correctly without reference to the evolution. Ratber than appeal to the homotopy defined by the evolution of the time step, we handle these nodes in a somewhat ad hoc manner, using the experimental observation that the unphysical loops are usually very small. We shall argue though that the way we treat these nodes is reasonable. 
We now describe the algorithm in more detail. A list of the $\mathrm{NH}$ nodes inserted at the cross points is generated. The list also contains the (four) curves connected to each node, arranged in angular order, and an index for each of the sectors at the node bounded by the curves. A first loop over the NH node list applies observations $\mathrm{O} 3$ and $\mathrm{O} 4$ to identify physical areas, and sets the index for such areas. For those nodes that have three "physical" sectors the fourth must be unphysical; on a second loop the curves bounding that sector are deleted from the interface $I^{l}$ (and from the $\mathrm{NH}$ list), and the node removed from the $\mathrm{NH}$ node list. Since the same sector can be adjacent to two NH nodes (observation O5b), it is possible to now encounter $\mathrm{NH}$ nodes in the list with only two curves, which now must represent physical curves. We therefore check for such nodes in the loop, and delete them from the NH node list. This second loop is implemented recursively, finishing when no $\mathrm{NH}$ nodes with three identified physical sectors can be found. There are generally only a few cross points at each time step, so efficiency in this part of the algorithm is not an important consideration. This completes the first part of the algorithm. The remaining entries in the NH node list now have two or more sectors that satisfy observation O5.

In the second part of the algorithm we find the area of each sector that has not been marked as physical in the first part of the code. We then rely on the observation (based upon numerical experiment) that the sector with the smallest area is usually the unphysical sector. The application of this observation is tempered and supplemented by topological restrictions such as the requirement that the left and right components of the remaining sectors be consistent with those of near by curves. It is obviously possible to improve this part of the algorithm somewhat, in particular by making sure that any sector that is identified as the one with smallest area at a particular $\mathrm{NH}$ node also be the sector of smallest area at any other $\mathrm{NH}$ node to which it may belong. To achieve greater improvement in this part of the algorithm, it is necessary to refer to the topology of the interface $I^{0}$, Even in doing so, there may still be cases that can only be resolved by "common sense". Fig. 2 demonstrates one such case. The curve marked $A$ can be thought of as a vortex sheet, and the curve marked $B$ as enclosing a blob of different material. The dotted line marks the position of $B$ with respect to $A$ at the next time step. It is obviously impossible, by topological arguments referring only to $I^{0}$ and $I^{1}$, to distinguish between paths (a) or (b) and hence whether area $\mathrm{x}$ or $\mathrm{y}$ represents the physically impossible area. Only by knowledge of the entire homotopy $0<t<1$ (rather than just its two endpoints) is this possible. This however presents a burdensome numerical task in general; our belief is that the smallest area criterion is a reasonable choice, since if there is any doubt about which loop should be deleted, the best procedure is to repeat the time step with a smaller time increment. 
In the problems for which this untangling algorithm has been used the interface curves do not carry essential physical variables that are not also representable by the interior degrees of freedom. Thus for any given discontinuity in the solution wave, there is an option as to whether it will be tracked or not. In the present code, we have chosen to delete from the tangled $I^{1}$ interface the curves surrounding the unphysical sectors that result from the crossing of one curve in $\Gamma^{0}$ with another. For material boundaries between distinct fluids, this amounts to the deletion of thin filaments. For some problems it might be advantageous or even necessary to retain these filaments as a single tracked curve. (See Fig. 3) For example if the curves represent vortex sheets in an inviscid fluid and if the fluid in the interior (away from the curves) is constrained to be irrotational, then a single curve is needed to represent the net vorticity of the two curves that have collided. The modification of the algorithm to handle such cases is obviously a minor one.

\section{Examples}

The purpose of the untangling algorithm presented here is to extend the application of front tracking codes to the solution of "messy, practical" problems. We demonstrate this capability by an example involving repeated tracked wave interactions. The examples shown are taken from a study of the performance of various well arrangements for secondary oil recovery. Figure 4 shows the evolution of the oil-water front for a line drive geometry where the middle injection well has been displaced from the line set by the other four injection wells. The set of runs from which this example is taken were made to investigate the effect of such a perturbation in a homogeneous reservoir for various viscosity ratios. When the frontal mobility ratio is sufficiently high the offset of the well is amplified, and the middle well breaks through before the others. In the run presented here, the frontal mobility ratio $\mathbf{M}$ (for the forward displacement of oil by water) satisfies the stability bound $\mathbf{M}<1$. The injected fluid (water) is therefore less mobile than the surrounding fluid (oil). Although the expanding water fronts move laterally, and eventually merge, they do not completely block the flow of the more mobile oil from behind the injection wells, which is drawn into narrow channels between the water banks. The results suggest that these channels are unstable, with pinch off instability as the predominant mode, in conjunction with a weaker meandering "garden bose" instability.

The four figures shown in Fig. 4 are the positions of the water bank at times ranging from the time shortly after the first merging of fronts from the different injection sites has taken place (a) through the time when the middle well has broken through (d). These figures were selected such that one can trace the evolution of oll "bubbles" as they form (b), move toward the leading stable front (c), and hreak through (d), at which time (in this particular 
example) new "bubbles" are again forming.

Instabilities of this type for a finger of viscous fluid moving through a fluid of different viscosity have been observed previously in the numerical study of a flow of two fluids in a Hele-Shaw cell $[7,8]$, and are also seen experimentally (for recent experiments see e.g. [14]). Phenomena with a related appearance occur in the instability and breakup of jets in compressible gas dynamics.

There are two noteworthy differences between the model studied here, and the HeleShaw model. In the present simulation, no physical length scale comparable with the role played by surface tension in $[7,8]$, has been introduced to break the self similarity of the governing equations at small length scales. Therefore the smallest resolved distance (in this case the grid spacing) plays the role of a stabilizing mechanism at small length scales.

Fig. 5 shows the results of a 4 well line drive study with the inter-well distances arranged to conform with that of Fig. 4. The frontal mobility ratio of this series of runs.is slightly different from Fig. 4 (0.288 compared with .586 for Fig. 4). The permeability functions and connate data for this run were taken from a particular reservoir field. The three plots show the flow computed at the same time value under three mesh regimes a) $5 \times 20$ hyperbolic $8 \times 30$ elliptic b) $10 \times 40$ hyperbolic $15 \times 60$ elliptic c) $20 \times 80$ hyperbolic $30 \times 120$ elliptic. Under grid refinement we indeed find that while the overall evolution is the same, the bubbles become smaller and the time of their first appearance in the computation occurs later than in coarser grid calculations (This later conclusion is not evident from Fig. 5 but can be seen by comparing earlier times in the calculations on the three meshes.)

For realistic reservoir parameters and grid spacings the capillary pressure length scale, which breaks scale invariance, can be less than one grid spacing and so grid regularization of this small length scale phenomena is appropriate. The gr:d spacing also sets a length scale for numerically induced beterogeneity, to provide a seed for initialization of the instability. Thus while we believe that the correct zero-size-mesh limit for the equation with no physical cut-off of short distance bebavior is an infinitely thin oil channel separating the water banks, we feel the numerical grid regularization gives a more correct representation of a flow that would result with a physical small scale cut-off and heterogeneity: namely the appearance of pinch of $f$ and "garden hose" instabilities in this channel.

The second difference is the rarefaction region behind the shock in the model for immiscible displacement calculated here, as compared with only a contact surface in the Hele-Shaw model. The bubbles must traverse this rarefaction region as they move towards the leading water bank. The composition of the bubble will thus change during this traversal. Both the pinch off (bubble formation) and the meandering of the jet cause a significant 
amount of backflow and hysteresis in the region of the jet between the main fingers. Backflow and hysteresis in turn, lead to a rarefaction fan ahead of the water bank in the channel. In Fig. 6 the saturation contours for the last frame in Fig. 4 are plotted. The contour lines depict 0.1 increments in the saturation. Saturation values below 0.707 result from this backflow and hysteresis. Thus we see that the two dimensional jet instability provides a mixing mechanism which should be studied in more detail.

Our last figures are taken from a study of more typical reservoir well placements and contains 31 injection and production wells. The fronts and well positions, early in the calculations are shown in Fig. 7(a) and the position of the oil-water front when the last production well has broken through is shown in Fig. 7(b). The untangling algorithm handles the merging of the banks formed from water injected from the various wells, as well as the complicated case where many banks break through at the same well.

\section{Conclusion}

A list of six main problem areas for front tracking has been previously mentioned [4]:

1. Achieving second-order accuracy at a shock without post-shock oscillations.

2. Avoiding stringent limits on the time step arising in the bits and pieces of zones which are crossed by a tracked front.

3. Properly treating slip along a front.

4. Treating

a) highly distorted fronts and

b) changes of the topology of regions bounded by fronts from simply connected to multiply connected regions.

5. Treating collisions and intersections of fronts with other fronts and with boundaries.

6. Treating the disappearance of weakening fronts and the appearance of new fronts at boundaries or at collisions of other fronts.

The status of front tracking on these six difficulties is now as follows:

1. This goal requires an upgrade in the riemann solver and in the front coupling to the interior to be second order accurate.

2. This problem has been solved, but the related problem of optimal front-interior coupling deserves further study.

3, 4a. These problems are fully solved.

$4 b, 5$. The examples given in this article demonstrate the capability of the untangling algorithm presented here to handle complex interface interactions.

6. Work is in progress on these problems. What is required is the implementation of shock capturing methods and two dimensional Riemann problem solvers [15]. 


\section{References}

1. B. Bukiet, C. L. Gardner, J. Glimm, J. Grove, J. Jones, O. McBryan, R. Menikoff, and D. H. Sharp, "Applications of Front Tracking to Combustion, Surface Instabilities and Two-Dimensional Riemann Problems," ARO Conference Proceedings.

2. James Glimm and D. H. Sharp, "Numerical Analysis and the Scientific Method," $D O E$ Research and Development Report DOE/ER/03077-270, 1986.

3. J. Glimm, B. Lindquist, O. McBryan, and L. Padmanabhan, "A Front Tracking Reservoir Simulator I: The Water Coning Problem," in Frontiers in Applied Mathematics, vol. 1, SIAM, Philadelphia, 1983.

4. I-L. Chern, J. Glimm, O. McBryan, B. Plohr, and S. Yaniv, "Front Tracking for Gas Dynamics," J. Comp. Phys., vol. 62, pp. 83-110, 1986.

5. J. Glimm, W. B. Lindquist, O. McBryan, and G. Tryggvason, "Sharp and Diffuse Fronts in Oil Reservoirs: Front Tracking and Capillarity," SIAM, Proc. Math. and Comp. Methods in Seismic Exploration and Reservoir Modelling, Houston, Jan, 1985.

6. M. J. King, W. B. Lindquist, and L. Reyna, "Stability of Two Dimensional Immiscible Flow to Viscous Fingering," DOE Research and Development Report DOE/ER/03077-244, March, 1985.

7. G. Tryggvason and H. Aref, "Numerical Experiments on Hele-Shaw Flow with a Sharp Interface," J. Fluid Mech., vol. 154, pp. 1-30, 1983.

8. G. Tryggvason and H. Aref, "Finger Interaction Mechanisms in Stratified Hele-Shaw Flow," J. Fluid Mech., vol. 154, pp. 287-301, 1985.

9. J. Glimm, E. Isaacson, D. Marchesin, and O. McBryan, "Front Tracking for Hyperbolic Systems," Adv. in Appl. Math., vol. 2, pp. 91-119, 1981.

10. J. M. Hyman, "Numerical Methods for Tracking Interfaces," Physica, vol. 12D, pp. $396-407$.

11. J. Glimm and O. McBryan, "A Computational Model for Interfaces," Adv. Appl. Math., vol. 6, pp. 422-435, 1985.

12. J. Glimm, C. Klingenberg, O. McBryan, B. Plohr, D. Sharp, and S. Yaniv, "Front Tracking and Two Dimensional Riemann Problems," Adv. in Appl. Math., vol. 6, pp. $259-290,1985$.

13. O. McBryan, "Elliptic and Hyperbolic Interface Refinement in Two Phase Flow," in Boundary and Interior Layers - Computational and Asymptotic Methods, ed. J. Miller, Boole Press, Dublin, 1980. 
14. J. V. Maher, "Development of Viscous Fingering Patterns," Phys. Rev. Lett., vol. 54, pp. 1498-1501, 1985.

15. W. B. Lindquist, "Construction of Solutions for Two Dimensional Riemann Problems," Adv. Hyp. PDE's., (To Appear). 


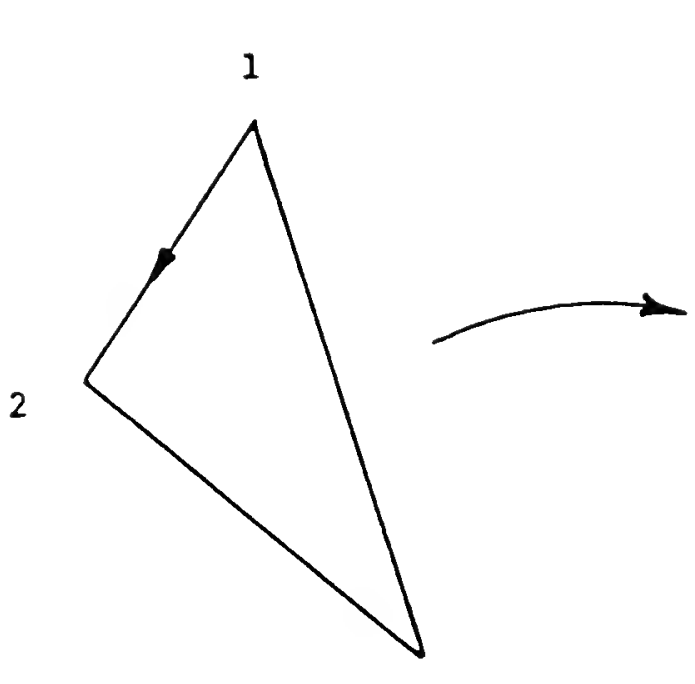

3

$I^{0}$

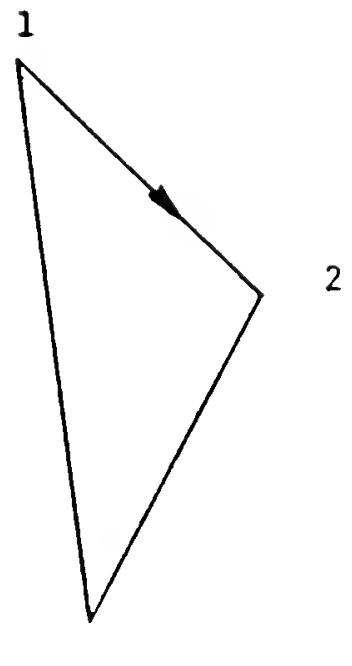

3

$I^{1}$

Fig. 1. An example of the inversion of a loop during propagation over a single time step. 
(a)

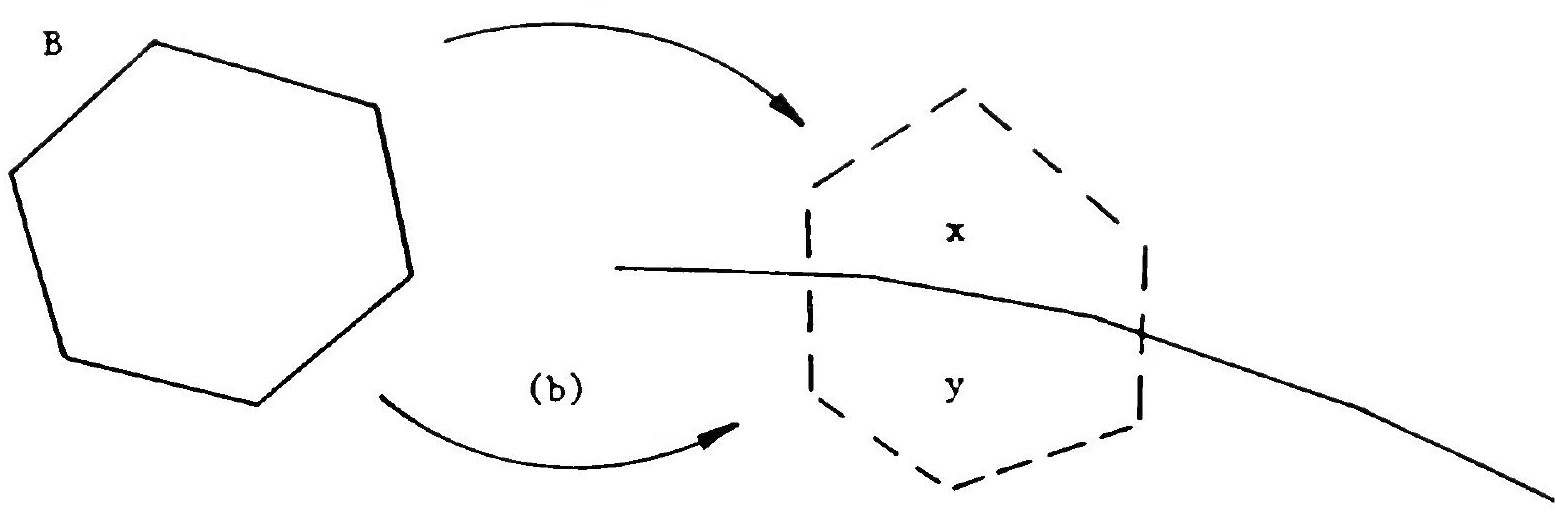

Fig. 2. A case where two curves cross each other in which it is impossible to determine the area which represents the the unphysical region from only the topology of the interface at $t^{0}$ or $t^{1}$. 


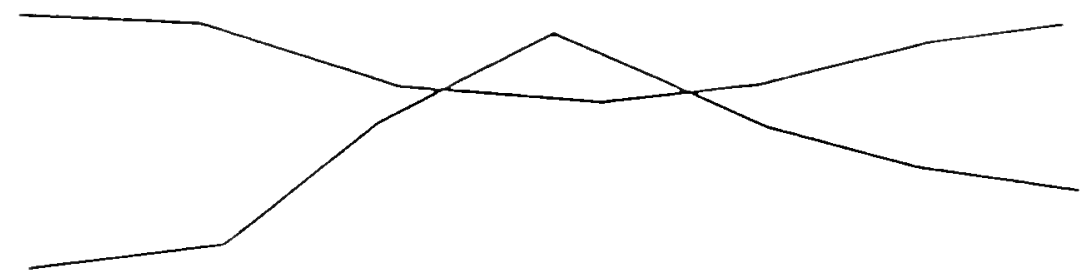

(a)
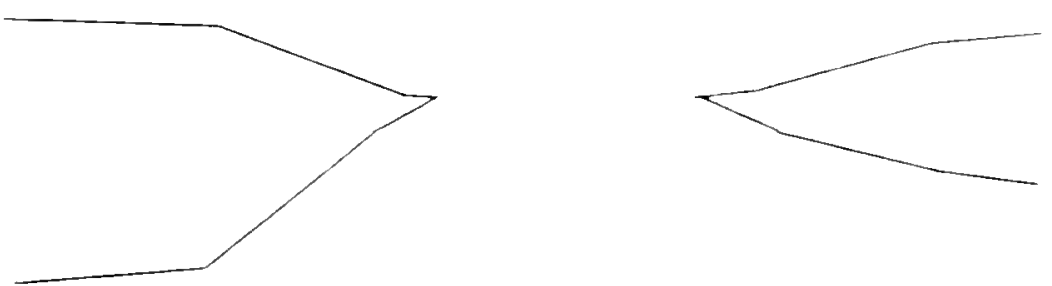

(b)

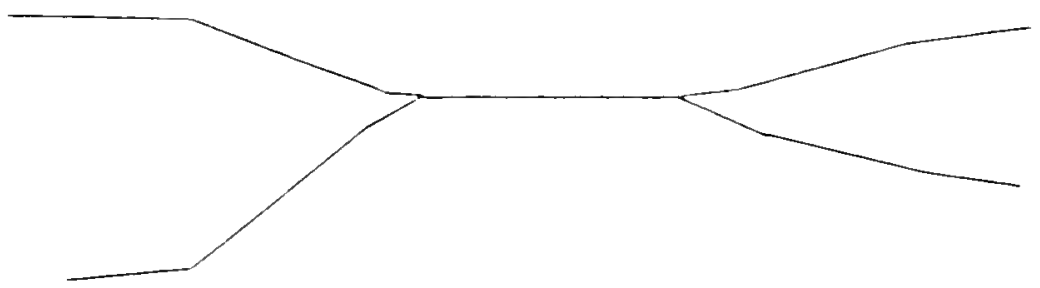

(c)

Fig. 3. a) A typical intersection of two curves which define the sides of a thin materiat channel. b) One method of resolving the resultant intersection. Here the channel is pinched off. This may result in convergence to an exact solution in an $L_{1}$ norm rather than $L_{x}$. c) A second method of resolution where the narrow channel is represented by a single curve. Method b) is currently implemented in the front tracking code described in the text. 


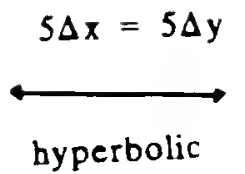

(a) Time step 22 .

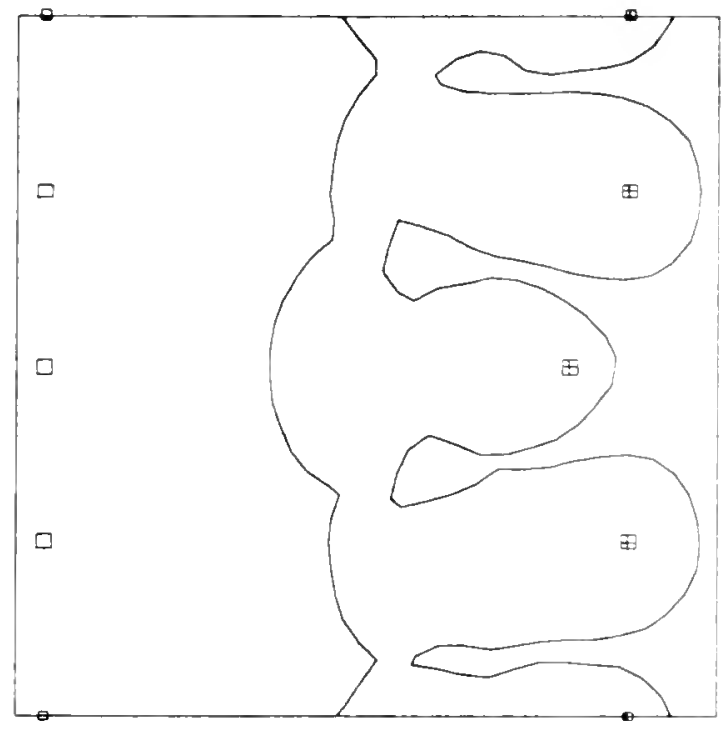

(c) Time step 36 .

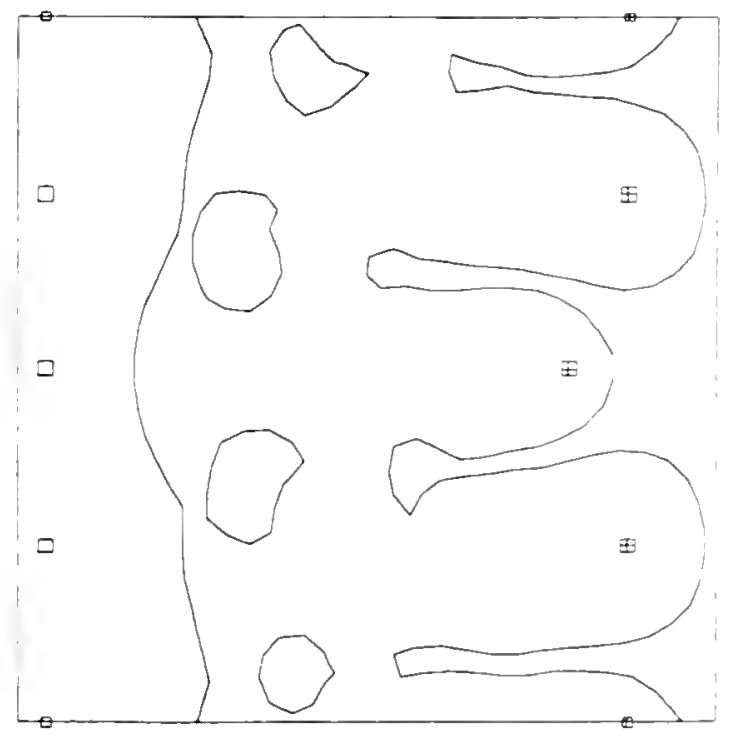

(b) Time step 29 .

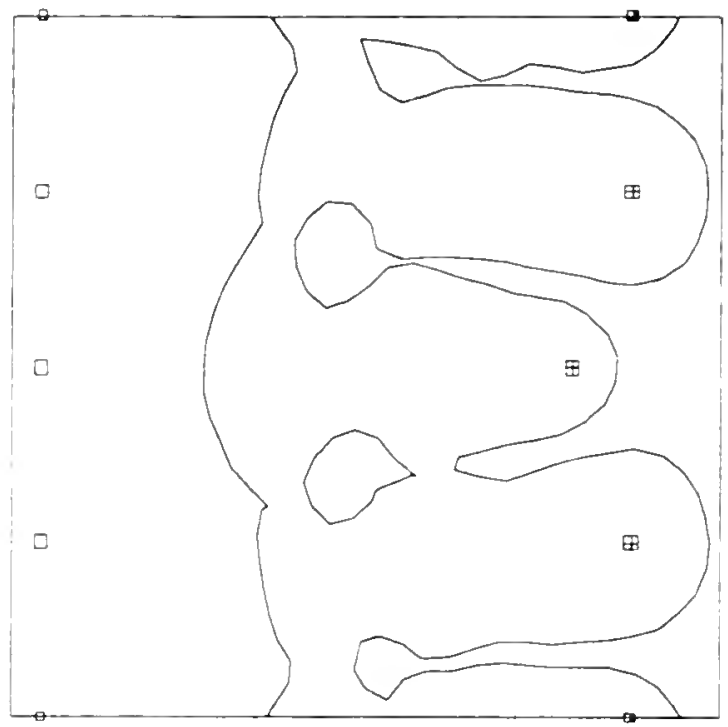

(d) Time step 44

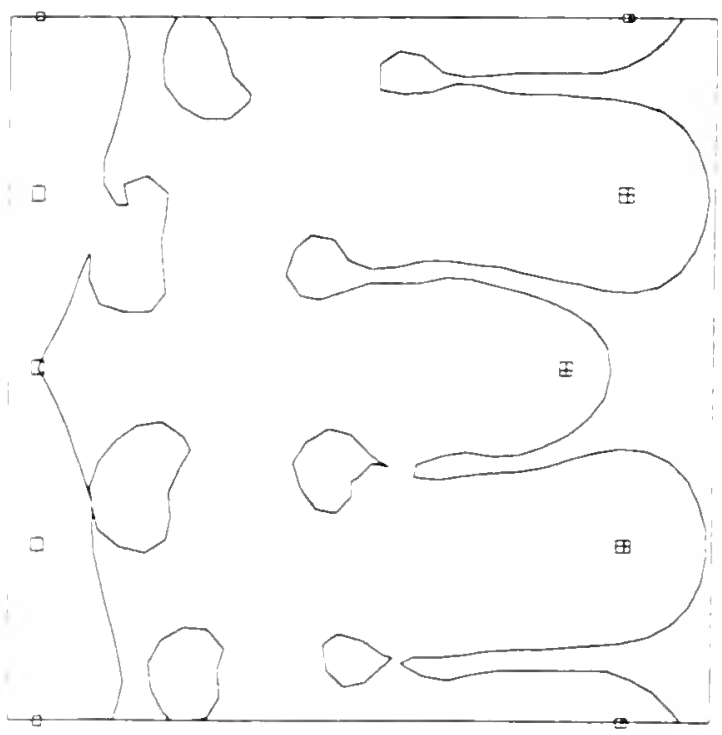

Fig. 4. An example of the complex interface interactions that can be handled by our untangling procedure: a line drive well configuration. The calculation is for immiscible displacement with quadratic permeability functions and equal viscosities of oil and water. The frontal mobility ratio for water displacing oil is 0.586 , the far field mobility ratio is 1.0 . The grid for the elliptic equations is 32 by 32 mesh blocks, for the hyperbolic 20 by 20 . 


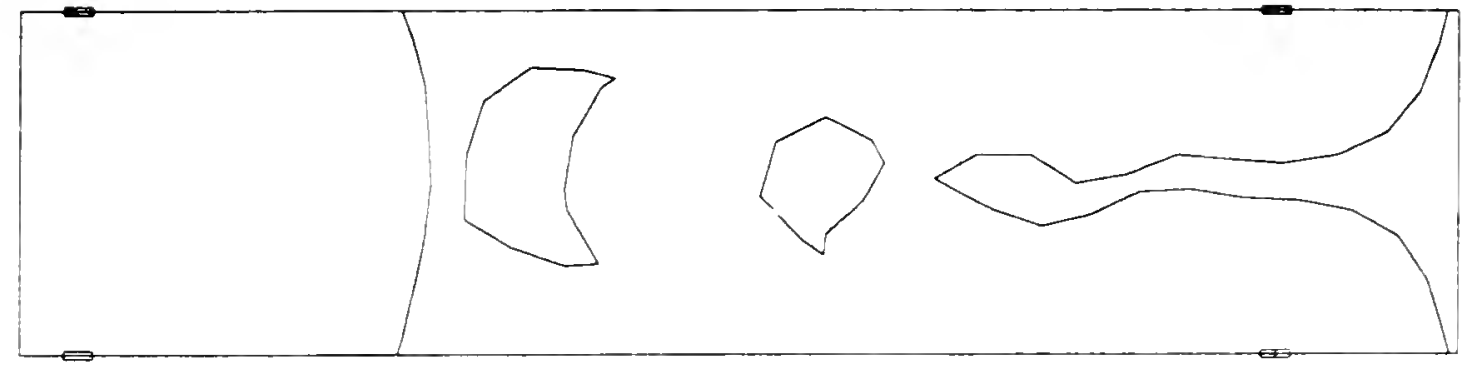

(a)

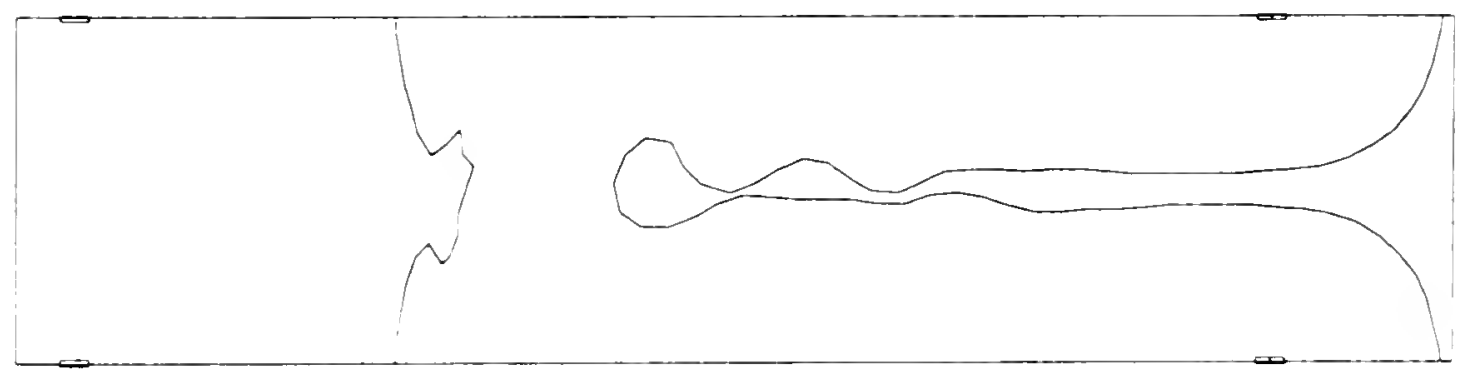

(b)

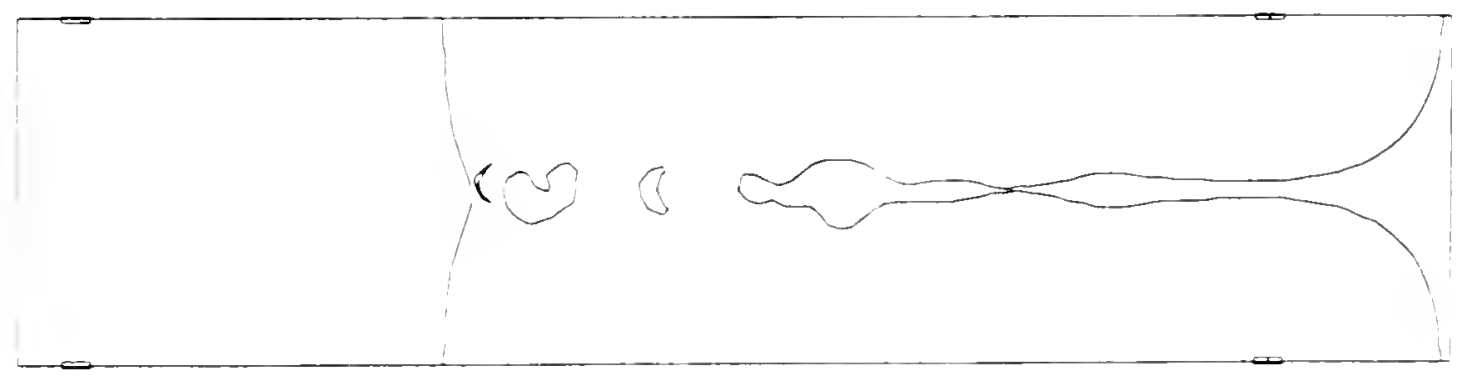

(c)

Fig. 5. A mesh refinement calculation of the unstable onl changel that forms between the merging water banks. Meshes used - a) $5 \times 20$ byperbolic, $8 \times 30$ elliptic b) $10 \times 40$ hyperbolic, $15 \times 60$ elliptic c) $20 \times 80$ hyperbolic, $30 \times 120$ elliptic 
$5 \Delta x=5 \Delta y$

$5 \Delta x=5 \Delta y$

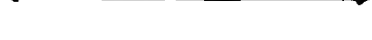

byperbolic

elliptic

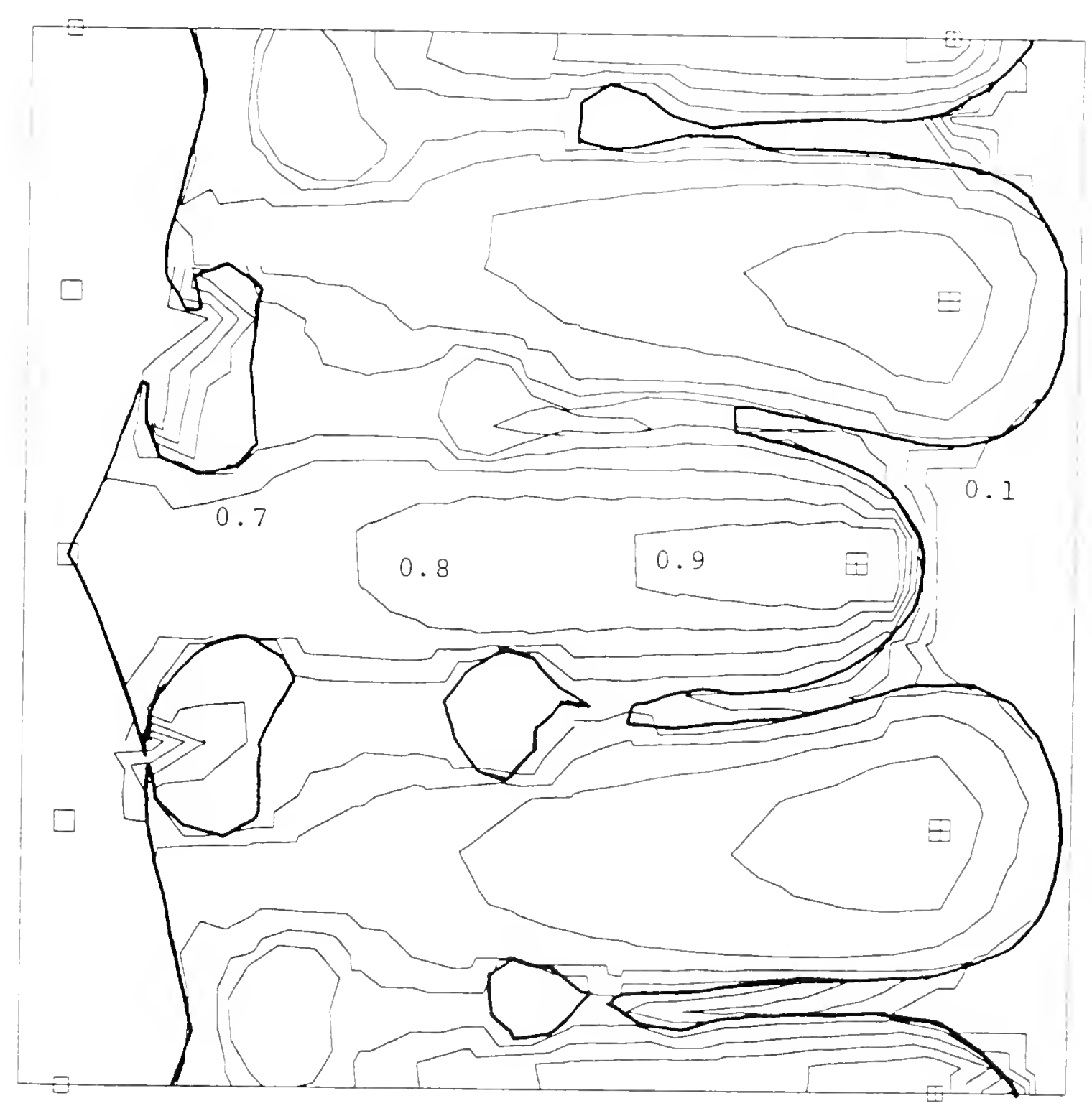

Fig. 6. Saturation contours in 0.1 increments for Fig. $4(d)$. Selected saturation contours are labeled. 
$10 \Delta x=10 \Delta y-18-$

hyperbolic
$10 \Delta x=10 \Delta y$

elliptic (a) Time step 0.

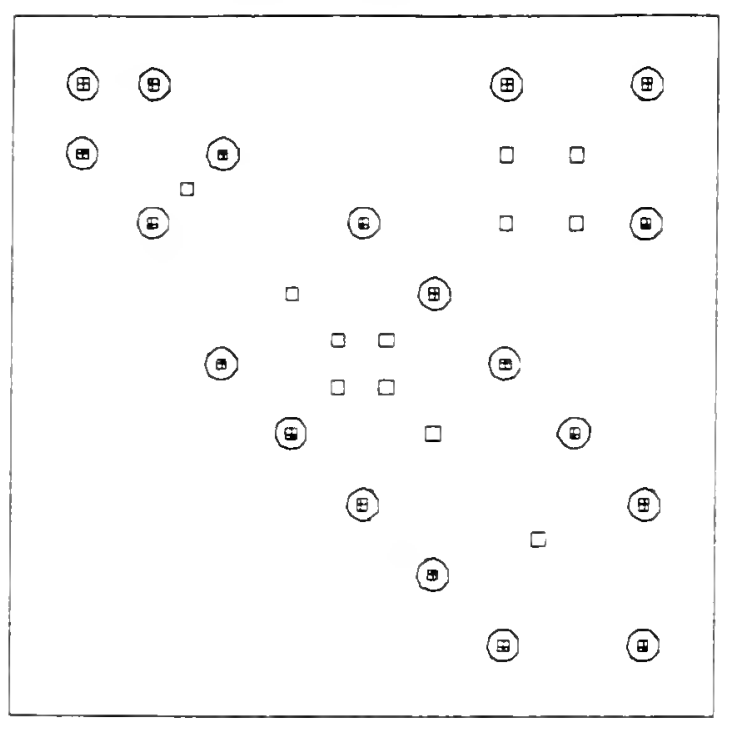

(b) Time step 40 .

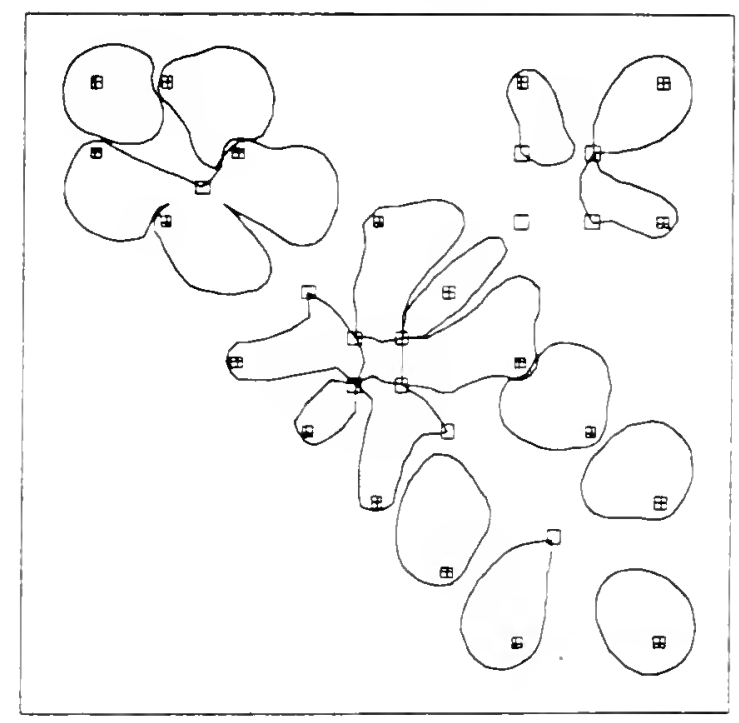

(c) Time step 80 .

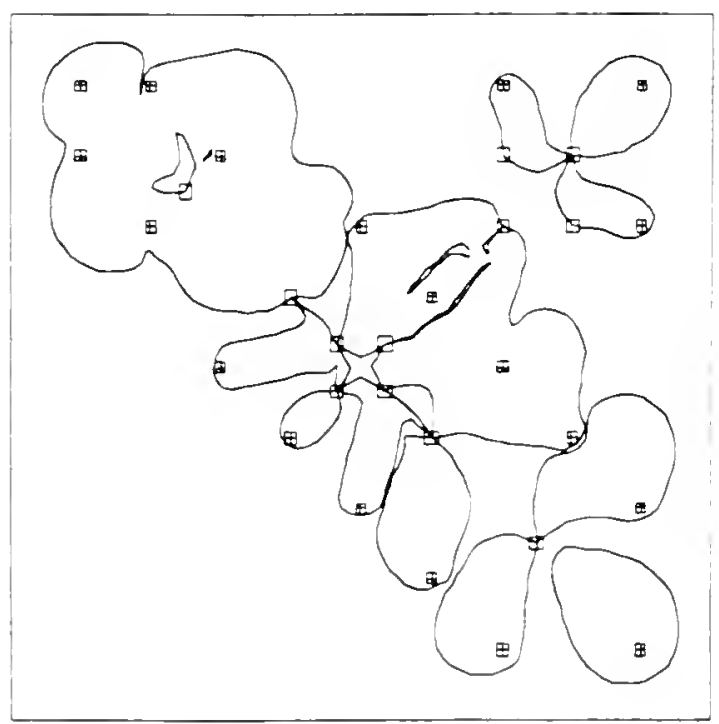

(d) Time step 240 .

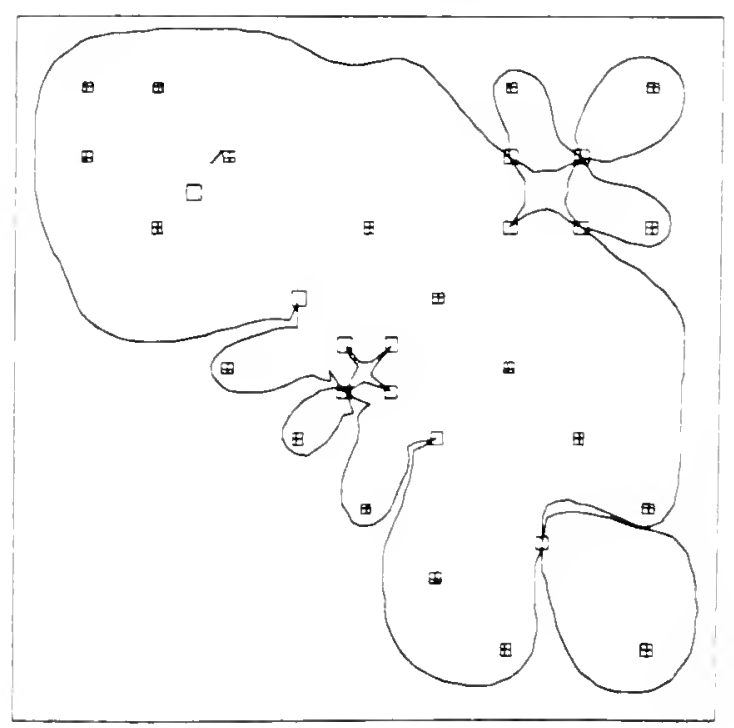

Fig. 7. Plots of the fronts for a more complicated well configuration consisting of 19 injecting wells (crossed squares) and 12 producing wells (open squares). The frontal mobility ratio for water displacing oil is 1.33 . The elliptic grid is 64 by 64 mesh blocks, while the hyperbolic grid is 48 by 48 mesh blocks. 
$5 d x-5 d y$

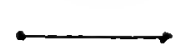

hyperbolic
$5 \Delta x-51 y$

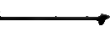

elliptic

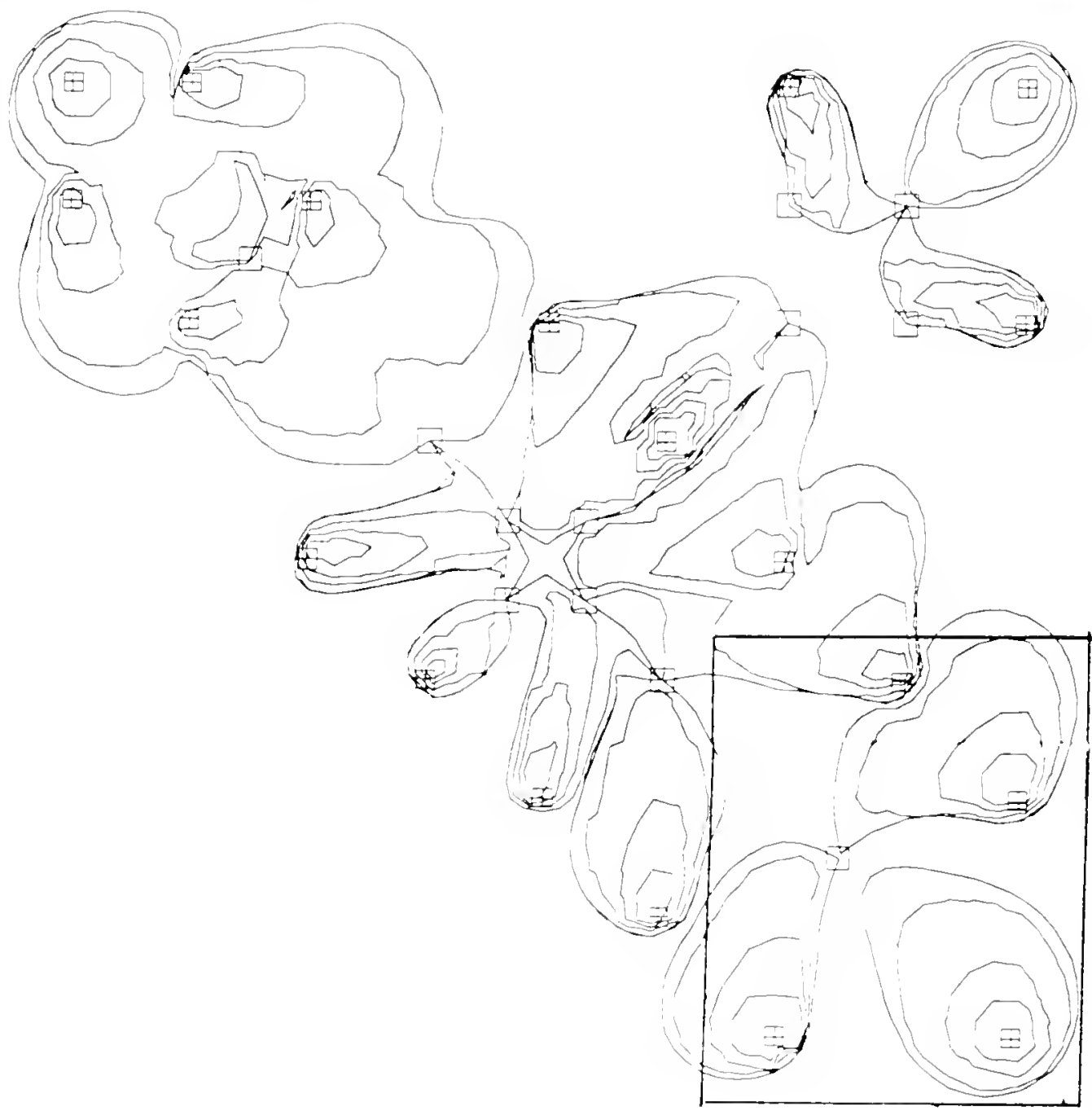

F:g. 3. Saturation contour plots for Fig. ${ }^{7}(\mathrm{c})$. The regron in the rectangle is blown up in the nexi rigure. 
$2 \Delta x=2 \Delta y$

$$
2 \Delta x=2 \Delta y
$$

hyperbolic

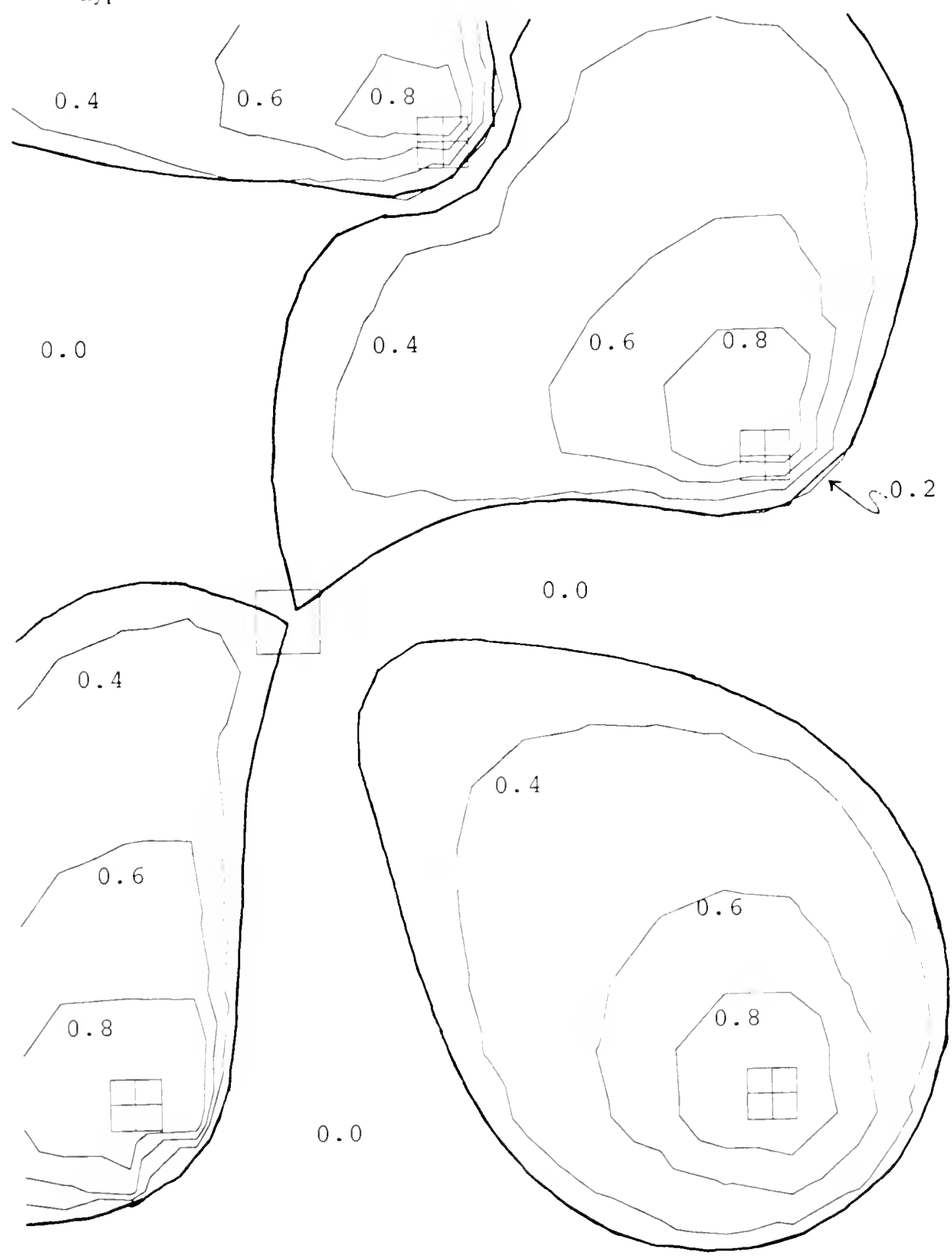

Fig. 9. Blowup of a subregion in Fig 8 . The $s=.2$ contour results from backflow. There is no numerical diffusion across the tracked front. The tracked front is drawn as a heavier line than the saturation contours. 
NYU DOE/ER 03077-272

Gl imm, James

The bifurcation of tracked scalar waves.

c. 1

This book may be kept

\section{FOURTEEN DAYS}

A fine will be charged for each day the bonk is kept overtime

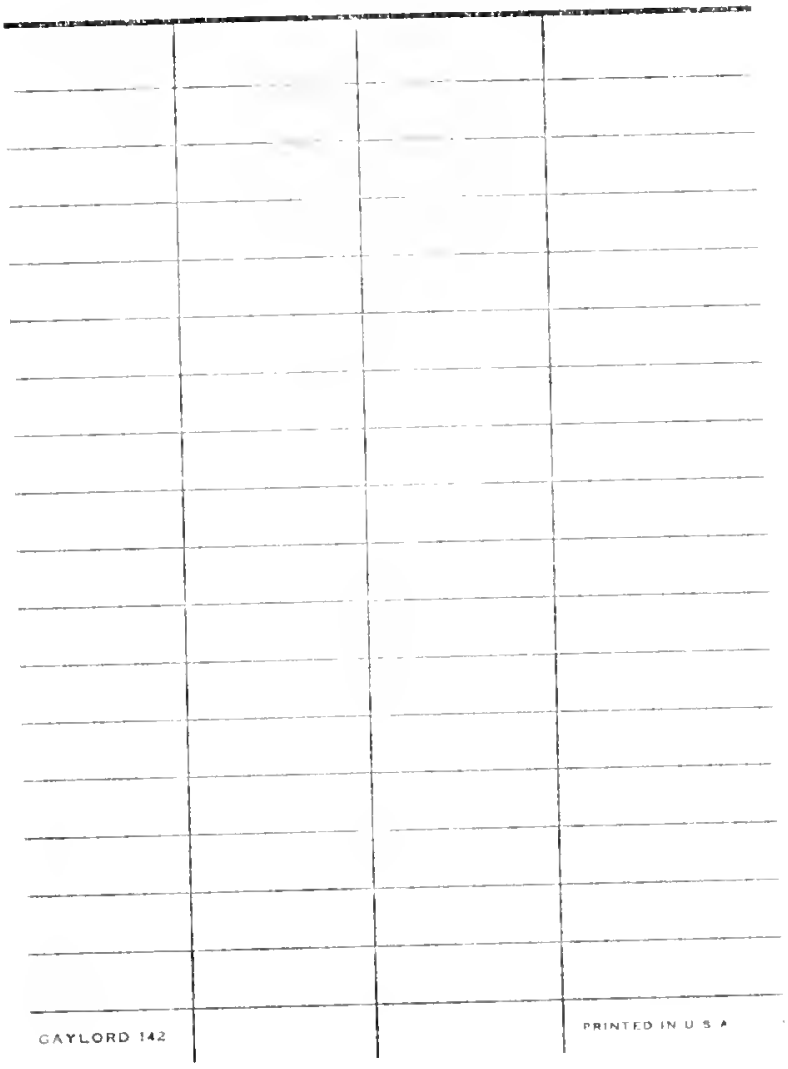

\section{LIBRARY}

N.Y.U. Courant Institute of Mathematical Sciences

251 Mercer St.

New York, N. Y. 10012 
